PAPER

\title{
Targeting the subthalamic nucleus for deep brain stimulation: technical approach and fusion of pre- and postoperative MR images to define accuracy of lead placement
}

\author{
N A Hamid, R D Mitchell, P Mocroft, G W M Westby, J Milner, H Pall
}

J Neurol Neurosurg Psychiatry 2005;76:409-414. doi: 10.1136/jnnp.2003.032029

See end of article for authors' affiliations .......................

Correspondence to: Mrs R D Mitchell, Department of Neurosurgery, Queen Elizabeth Medical Centre, University Hospital Birmingham, Birmingham, B15 2TH, UK.

Rosalind.Mitchell@ uhb.nhs.uk

Received

17 November 2003

In revised form 6 May 2004

Accepted 9 June 2004

\begin{abstract}
Objectives: To define the role of magnetic resonance imaging (MRI) and intraoperative electrophysiological recording in targeting the subthalamic nucleus (STN) in Parkinson's disease and to determine accuracy of electrode placement.

Patients and methods: We implanted 54 electrodes into the STN in 27 patients. Target planning was done by coordinate guidelines and visualising the STN on MRI and defined in relation to the mid-point of the AC-PC line. Intraoperative microelectrode recording was used. We adjusted electrode positions for placement in the centre of the STN electrical activity and verified this on postoperative MRI in 16 cases, which were fused to the preoperative images to measure actual error in electrode placement in the three axes.

Results: Based on coordinate calculation and MRI localisation, the mean of the target was $11.5 \mathrm{~mm}$ lateral, $2.5 \mathrm{~mm}$ posterior and $4.1 \mathrm{~mm}$ inferior to the mid-point of the AC-PC line. Fifty good electrophysiological recordings of the STN (average length $4.65 \mathrm{~mm}$ ) were achieved and target point adjusted in $90 \%$ of lead placements. The mean of the final target after electrophysiological correction was $11.7 \mathrm{~mm}$ lateral, $2.1 \mathrm{~mm}$ posterior, and $3.8 \mathrm{~mm}$ inferior to the mid-point. The distance from the centre of the electrode artefact to the final target used after electrophysiological recording on the fused images was $0.48 \mathrm{~mm}, 0.69 \mathrm{~mm}$, and $2.9 \mathrm{~mm}$ in the $x, y$, and $z$ axes, respectively. No postoperative MRI related complication was observed.

Conclusion: Both direct visualisation of the STN on MRI and intraoperative electrophysiological recording are important in defining the best target. Individual variations exist in the location of the STN target. Fewer tracks were required to define STN activity on the side operated first. Our current stereotactic method of electrode placement is relatively accurate.
\end{abstract}

$\mathrm{S}$ ince the pioneering work of Benabid et al ${ }^{1}$ and Pollak et $a l^{2}$ in 1993-1994, chronic high frequency stimulation of the subthalamic nucleus (STN) has become the preferred surgical treatment for medically refractory Parkinson's disease..$^{3-5}$ The STN gained popularity as a potential target for treatment of Parkinson's disease in 1990 when Bergman et $a l^{6}$ proved experimentally that a lesion in the motor part of the STN in a non-human primate model reversed the symptoms of Parkinson's disease.

The degree of clinical improvement achieved by deep brain stimulation (DBS) is largely dependent on the accuracy of lead placement..$^{7-9}$ Various methods have been used to localise the STN. One of the methods involves using an atlas of brain structures and pre-existing data obtained by workers with considerable experience in the field of STN stimulation. ${ }^{10}$ The coordinates thus obtained are referenced to a line drawn from the anterior commissure (AC) to the posterior commissure (PC). This can be defined using stereotactic ventriculography or three dimensional magnetic resonance imaging (MRI). However, as this method does not take into account individual variations in STN location, direct visualisation of the STN on special MRI sequences is a suitable method for accurate localisation. ${ }^{3}$ Using microelectrodes, electrophysiological recording of the STN can be obtained during the operation. This provides real time physiological confirmation of the target and is felt to be a very useful adjunct to current advanced brain imaging techniques and target localisation by many workers. ${ }^{10-12}$ A hybrid technique taking into consideration both the methods outlined above for STN localisation has been reported to be useful strategy. ${ }^{3}$

As the STN is a relatively small structure estimated at $9 \times 7 \times 5 \mathrm{~mm},{ }^{13}{ }^{14}$ it is important to define the exact location of the electrode being used for stimulation. Postoperative documentation of actual electrode location is important to verify the accuracy of the surgical technique being used and to define the area being stimulated. This knowledge when studied in conjunction with clinical response will prove vital in determining the best area for stimulation in Parkinson's disease. In the present study we aimed to evaluate the role of MRI and intraoperative electrophysiological recording in targeting the STN in patients with Parkinson's disease and determine the accuracy of electrode placement.

\section{PATIENTS AND METHODS \\ Patients}

Between June 2000 and June 2003, 54 DBS electrodes were implanted in 27 patients with Parkinson's disease ( 16 men: average age 55 years, range 41-66; 11 women: average age 58 years, range 49-69). All patients had bilateral implantation at the same operation.

Abbreviations: $\mathrm{AC}$, anterior commisure; DBS, deep brain stimulation; MRI, magnetic resonance imaging; PC, posterior commisure; STN, subthalamic nucleus 
All patients were seen jointly by a neurosurgeon and a neurologist and the diagnosis of Parkinson's disease was confirmed by more than one consultant neurologist. Ongoing good response to dopaminergic treatment was seen in all patients and only those patients who had medically refractory motor fluctuations were offered surgery. All patients underwent psychometric testing before the operation. Informed consent was obtained from all patients.

\section{Surgical procedure}

A Leksell-G stereotactic frame was applied and patients were taken to the MRI suite (Siemens Magnetom Vision, Siemens AG, Medical Solutions, Erlangen, Germany), where special sequences were obtained on a 1.5 Tesla machine. Magnetisation prepared rapid acquisition gradient echo (MPRAGE) sequences were obtained (slab thickness $240 \mathrm{~mm}$ (effective thickness $2.0 \mathrm{~mm}$ ), matrix $256 \times 256$, TR 9.7) and the scanning time was six minutes and 30 seconds. The MPRAGE (3D acquisition) sequence was used for calculations. To better define the STN, T2-weighted images (TR 2800, TE 90 , flip angle $90^{\circ}$, slice thickness $2.0 \mathrm{~mm}$ ) were obtained. This sequence took six minutes and 47 seconds (see fig 1 for MRI showing the deep nuclei). These parameters were used in the last 21 patients of the series as they were seen to define the STN more clearly. In the first six patients, a different sequence was used (TR 6612, TE 112, flip angle $180^{\circ}$, slice thickness $3 \mathrm{~mm}$ ).

All images were transferred to the BrainLAB workstation and projected in all three dimensions (axial, coronal, and sagittal). The AC and the PC were marked and the centre of the AC-PC line determined. For initial target localisation we employed the values suggested by Benabid et al for STN stimulation-that is, $12 \mathrm{~mm}$ lateral, $3 \mathrm{~mm}$ posterior and $4 \mathrm{~mm}$ inferior to the mid-point of the AC-PC line. In the first six patients these guidelines were followed with minor changes guided by assumed anatomical relations-for example, the third ventricle and the red nucleus. After that the MRI specifications for obtaining the T2-weighted MR images were changed to visualise the STN and other nuclei more clearly (see MRI specifications above). Because of the improved visualisation of the STN in the rest of the placements $(n=42)$, the initial target coordinates based on the values of Benabid et $a l^{9}$ were changed to get the best placement in the STN.

Single track microelectrode recordings (Intraoperative Microelectrode; Medtronic Ltd, Watford, UK) were obtained

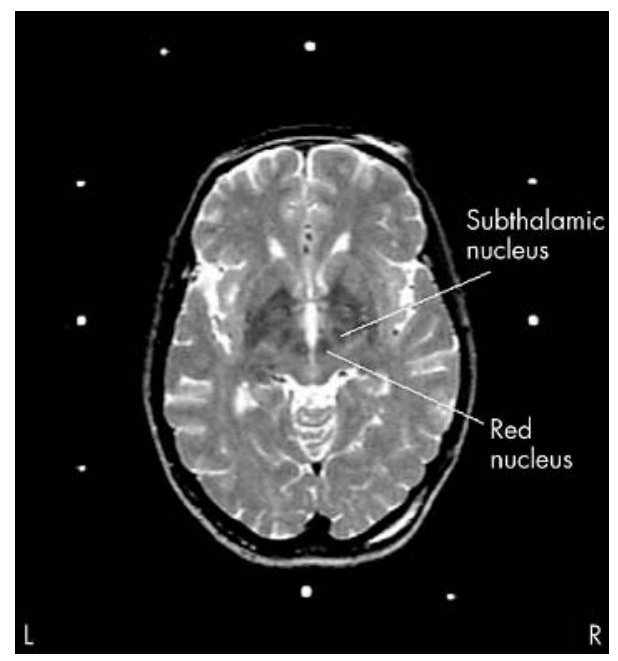

Figure 1 An axial T2-weighted image displaying the hypointense deep nuclei and the subthalamic nucleus. using Medtronic Leadpoint neural activity monitoring system. In the initial eight patients, recording was started $5 \mathrm{~mm}$ above the presumed target, but a new microdrive was used in the subsequent 19 patients and the recording was started $10 \mathrm{~mm}$ above and continued until no further STN activity was recorded (usually $5 \mathrm{~mm}$ below target). As the recording electrode was advanced, entry into the STN was identified by a sudden increase in the density of cellular discharge, with the characteristic irregular pattern of discharge-spikes of different sizes, occurring at random intervals (fig 2). On coming out of the STN a quiet period (background noise) was seen followed by recording from the substantia nigra if the recording was continued far enough, described as high frequency (50-60 spikes/s) discharge pattern. ${ }^{11}$ Characteristic STN recordings (visual and audio) were identified and the depth of the STN activity was noted. Identification of STN activity was only based on the visual identification of the raw trace. Intraoperative stimulation was not used in this study. If a satisfactory recording was not obtained, the recording electrode was removed and reinserted $2 \mathrm{~mm}$ from the original target. The change in direction was determined after re-studying the MR images on the BrainLAB workstation. The next best target likely to pass through the STN was selected.

The centre of the point of best electrical activity was selected as the final target. The microelectrode was replaced with a permanent quadripolar macroelectrode (Medtronic electrode no. 3389) to target the centre of the STN electrical activity. The proximal part of this electrode consists of four nickel conductor wires insulated with a polytetrafluoroethylene jacket tubing. The distal part has four metallic noninsulated contacts of $1.5 \mathrm{~mm}$ spaced at $0.5 \mathrm{~mm}$ intervals. The diameter of the distal electrode is $1.27 \mathrm{~mm}$. Based on the clinical response any of the four contacts can be used for stimulation. In most cases lateral skull $x$ rays were obtained at this point with image intensifier carefully positioned to locate the target point in the centre of the Leksell-G frame rings (fig 3). This was to check the depth of the electrode. In the first 18 patients, because of difficulties in securing the electrode leads in the burr hole, some movement in the electrode position was possible in the line of the trajectory; the $x$ rays were used to correct this movement. In the last nine patients, the new fixation device Navigus Cranial Base and Cap (Image-guided Neurologics, Melbourne, FL) was used. Finally under general anaesthesia, the wires were tunnelled and the stimulator (Kinetra; Medtronic Ltd) placed subcutaneously in the chest wall.

\section{Postoperative MRI and fusion of images}

In the first consecutive 16 patients, postoperative MRI was done routinely. After reviewing the satisfactory results of

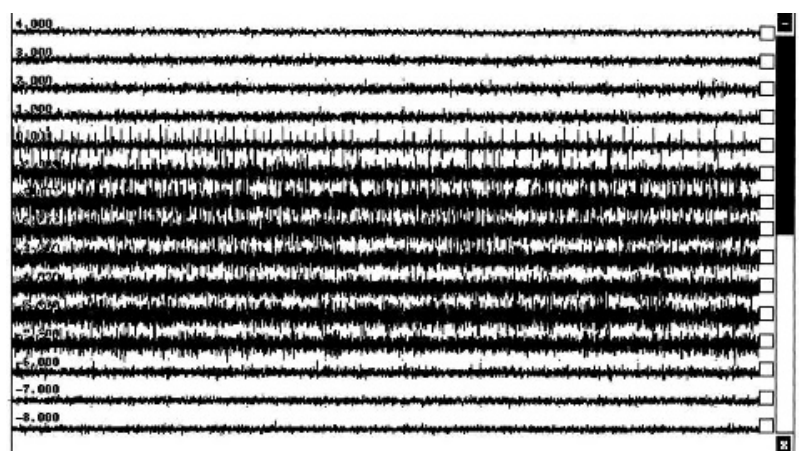

Figure 2 A typical STN recording $(-5.000 \mathrm{~mm}$ to $0.000 \mathrm{~mm}$ ) showing the irregular pattern of discharge with spikes of different sizes, occurring at random intervals. 


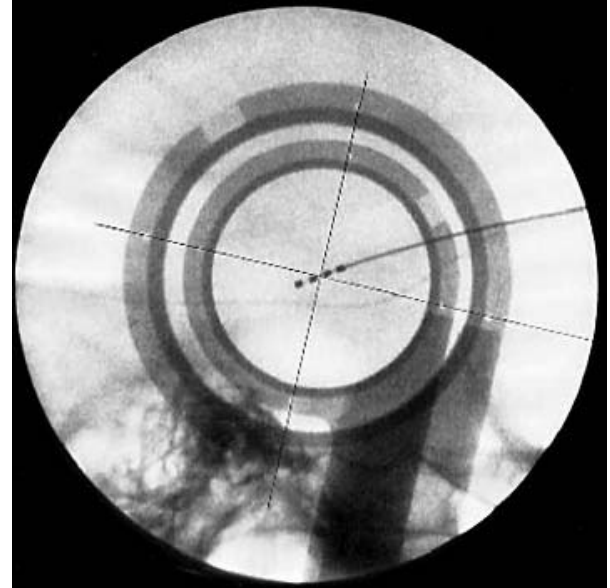

Figure 3 An intraoperative image intensifier picture, showing the centre of the electrode array at the target point (in the centre of the rings of the Leksell-G frame).

these 16 patients, routine postoperative MRI was not done in the rest of the patients. A strict protocol was followed for the MRI: only head coils were used in a 1.5 Tesla magnet and patients were observed during and after the procedure. MRI sequences for postoperative imaging were similar to the preoperative imaging (MPRAGE; slab thickness $240 \mathrm{~mm}$ (effective thickness $2.0 \mathrm{~mm}$ ), matrix $256 \times 256$, TR 9.7). Using a commercially available rigid body fusion programme that autofuses images by matching voxel for voxel (BrainLAB), pre- and postoperative images were fused to check the accuracy of lead placement by comparing actual lead position to the final target used after electrophysiological recording (fig 4).

The centre of the lead artefact was defined in the axial plane and the distance from this point to the final target used after electrophysiological recording was measured in the $\mathrm{x}$ (medial-lateral) and y (anterior-posterior) axes. This was the error of lead placement in the respective planes. For the $\mathrm{z}$ (superior-inferior) axis, the lowest point of the electrode artefact was identified. The centre of the electrode array, which should be at the final target point is $4.25 \mathrm{~mm}$ from this point. The distance between the centre of the electrode array and the final target used after electrophysiological recording was the error in the $\mathrm{z}$ axis.

\section{RESULTS}

\section{Target localisation: MRI}

The quoted figures for STN stimulation: $12 \mathrm{~mm}$ lateral, $3 \mathrm{~mm}$ posterior, and $4 \mathrm{~mm}$ inferior to the midpoint of the AC-PC line ${ }^{9}$ were used as an initial guideline only. In 21 patients (42 STN targets) where the STN was clearly visualised on
T2-weighted images the target was moved on the basis of this information. It was seen that the actual target movement was $1 \mathrm{~mm}$ or more in 20 target definitions in the $\mathrm{x}$ axis (48\%), 20 in the y axis (48\%), and 3 in the $z$ axis (7\%). This probably depicts the difference in individual anatomy. Based upon good visualisation of the STN on MRI in the last 42 electrode placements, the mean calculated target was 11.6 (SD 0.87) $\mathrm{mm}$ lateral, $2.3(0.85) \mathrm{mm}$ posterior, and $4(0.50) \mathrm{mm}$ inferior to the midpoint of the AC-PC line. Including the initial 12 placements, in a total of 54 placements, based on coordinate calculation and MRI localisation, the mean deviation of the target was 11.5 (1.18) mm lateral, $2.5(0.90) \mathrm{mm}$ posterior, and $4.1(0.53) \mathrm{mm}$ inferior to the mid-point of the AC-PC line.

\section{Intraoperative electrophysiology}

Intraoperative electrophysiological recordings for STN localisation were obtained in all patients. Good recordings were seen in 52 placements (96\%). In one patient, probably due to some mechanical problem with the equipment used, no characteristic recording was obtained despite using three different tracks on both sides. In another patient who had undergone a right sided pallidotomy in the past, STN activity was recorded for only $2 \mathrm{~mm}$ on the right side and $1 \mathrm{~mm}$ on the left side. Because of his previous lesion, it was thought that trying to get a better STN recording by making multiple tracks could cause more damage, so further tracks were not made. In the rest of the 50 recordings, the average length of the characteristic STN recording was $4.65 \mathrm{~mm}$ (range 3-7 mm). A typical recording is shown in fig 2 .

On the basis of these findings, in a total of 52 implantations ( the patient in whom the STN activity was not seen was excluded from the statistics of electrophysiological recording), the target was readjusted in 47 electrode placements $(90 \%)$ to lie at the centre of the STN activity for maximum accuracy. In 18 lead placements (35\%), the target point was moved by $2 \mathrm{~mm}$ or more compared with the MRI defined target point. The average movement in the $\mathrm{x}$ axis was $0.42 \mathrm{~mm}$, in the y axis $1.0 \mathrm{~mm}$, and in the $\mathrm{z}$ axis $0.88 \mathrm{~mm}$. However, the greatest movement was seen in the depth or trajectory of the electrode, which was an average of $1.19 \mathrm{~mm}$.

It should be appreciated that any movement in the line or trajectory of the electrode will have an effect on the position of the target point in the $\mathrm{x}, \mathrm{y}$, and $\mathrm{z}$ axes depending upon the angle of the trajectory in the coronal and the sagittal planes. It should also be noted that the actual trajectory being used is not in line with any of the three axes; so changing the depth of the electrode is not the same as changing the $\mathrm{z}$ axis. In this study, the mean angle of the trajectory was $17.5^{\circ}$ in the coronal plane and $38^{\circ}$ in the sagittal plane. Any movement in the depth of the electrode will have a small effect on the target position in $\mathrm{x}$ axis and a proportionately greater effect in the $y$ and $z$ axes. The above values depicting movement of the target position in the $\mathrm{x}, \mathrm{y}$, and $\mathrm{z}$ axes were calculated after
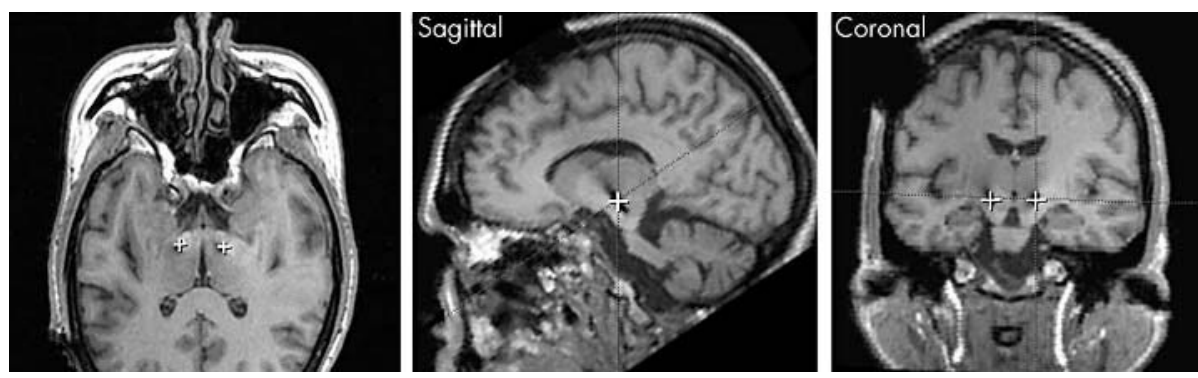

Figure 4 Fused images of pre- and postoperative MRI showing the final target used after electrophysiological recording (white crosses) superimposed over the electrode artefacts (black). 
making the necessary corrections taking into account the angles of the trajectories used.

\section{Tracks}

In 14 lead placements more that one track had to be made to get a better definition of the STN electrical activity. Two tracks were made in 12 placements, and three tracks in two placements. Among the 12 initial placements (six patients) where the STN was less clearly seen on MRI, more than one track was required in five placements $(42 \%$ of electrode placements in this group). In the subsequent 42 placements (21 patients) where special MRI sequences were used more effectively to define the anatomy, a second track was required in nine placements ( $21 \%$ of electrode placements).

STN recording was better on the side that was tested first, a second track being required in only $5 / 14$ cases. On the second side, 9/14 required more than one track for good STN recording (35\% and $64 \%$, respectively).

\section{Final target}

The mean of the final target that was used after electrophysiological recording was 11.7 (SD 1.30) $\mathrm{mm}$ lateral, $2.1(1.43) \mathrm{mm}$ posterior, and $3.8(1.22) \mathrm{mm}$ inferior to the centre of the AC-PC line (median: $12.0 \mathrm{~mm}, 2.30 \mathrm{~mm}$, and $3.8 \mathrm{~mm}$, respectively). Marked variation in these values was noticed among the patients in all the three planes (see fig $5 \mathrm{~A}$, $\mathrm{B}$, and $\mathrm{C}$ for the $\mathrm{x}, \mathrm{y}$, and $\mathrm{z}$ axes, respectively). The final quadripolar Medtronic electrode no. 3389 was placed at the defined target in such a way that in each individual placement the mid-point of the array was at the target.

\section{Postoperative MRI and fusion of images}

Postoperative MR images were fused with the preoperative MR images in 16 patients (see fig 4). In the axial images, the mean distance from the centre of the lead artefact to the final target corrected after microelectrode recording was $0.48(0.38) \mathrm{mm}$ in the $\mathrm{x}$ axis and $0.69(0.58) \mathrm{mm}$ in the $\mathrm{y}$ axis. For the $\mathrm{z}$ axis the lowest point of the electrode artefact in the sagittal and coronal planes was measured. From this point a value of $4.25 \mathrm{~mm}$ was subtracted to get the centre of the electrode array. The error was 2.9 (1.95) $\mathrm{mm}$ in the $\mathrm{z}$ axis. The electrodes were observed to lie lower than the final target used. As postoperative MRI and fusion of the images was done for only the initial 16 patients in whom the Navigus Cranial Base and Cap was not used for fixation, it is assumed that the possibility of this error has been reduced.

No complication related to postoperative MRI was observed. In this study only those cases who had STN implants were included, but it may be worth mentioning that a total of 35 patients with deep brain stimulators have undergone postoperative MR scans in our unit with no adverse effects.

\section{DISCUSSION}

\section{Target localisation: MRI}

Direct visualisation of the STN on T2-weighted fast spin echo sequences of MRI has been widely reported..$^{11}$ Many groups use a hybrid method of target localisation using direct visualisation of the STN and indirect coordinates based on the AC and PC relation. ${ }^{310} 16$ The point we used for initial target localisation was $12 \mathrm{~mm}$ lateral, $3 \mathrm{~mm}$ posterior, and $4 \mathrm{~mm}$ inferior to the AC-PC midpoint. Using direct visualisation of the STN in 42 lead placements on MRI, the target position was moved $1 \mathrm{~mm}$ or more in 20 target definitions in the $\mathrm{x}$ axis $(48 \%), 20$ in the $\mathrm{y}$ axis $(48 \%)$, and 3 in the $\mathrm{z}$ axis (7\%). In another study addressing this issue, more that $1 \mathrm{~mm}$ difference was seen in 34\% placements in the $\mathrm{x}$ axis and $70 \%$ in the $\mathrm{z}$ axis. ${ }^{3}$ In our study, on the basis of MR localisation of the STN in the last 42 placements, the mean of the target was
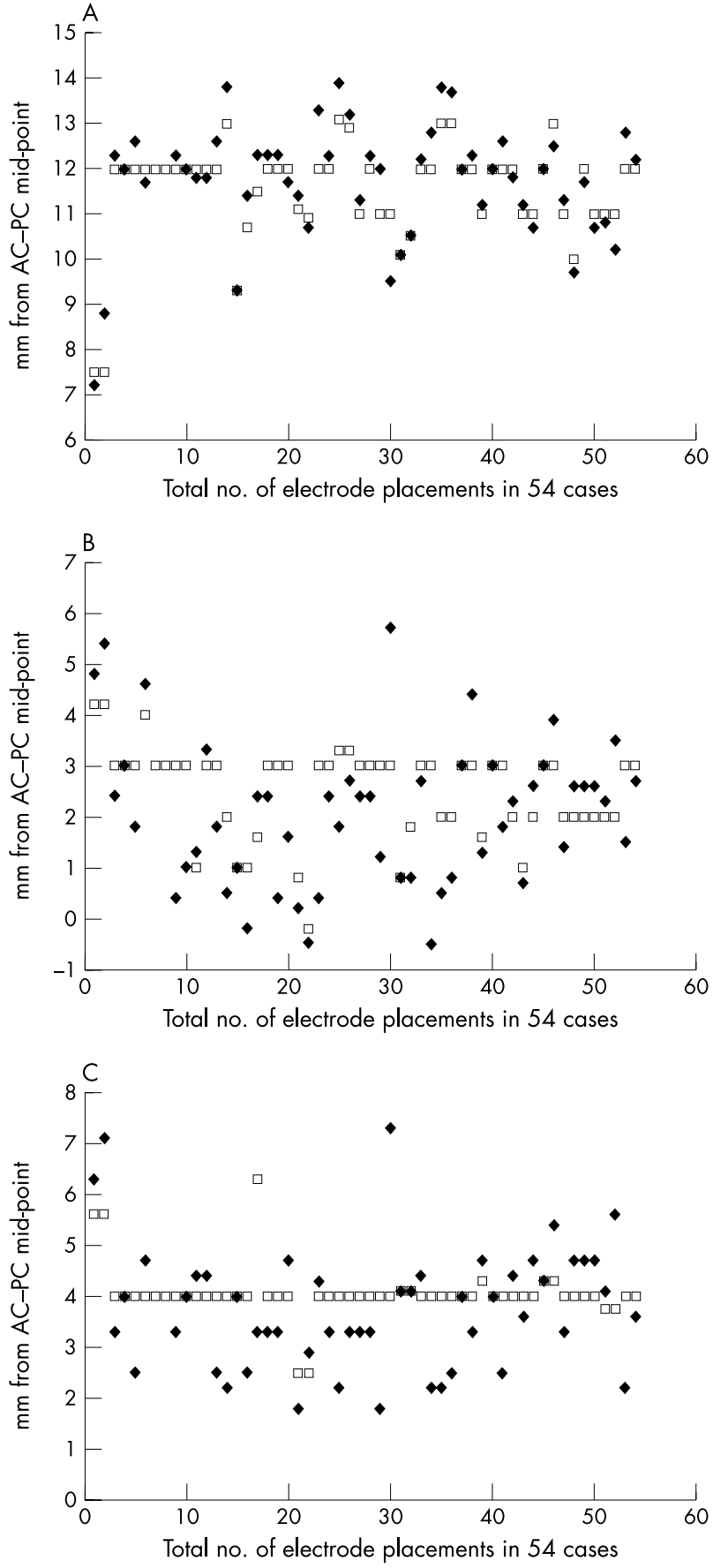

Figure 5 Target values in the (A) $x$ axis, $(B) y$ axis, and $(C) z$ axis in each of the 54 placements. The difference in the location of MRI defined targets (open squares) and the final targets after electrophysiological adjustment (filled diamonds) in each placement can be seen. The number of placements is in chronological order-that is, date of placement.

$11.6 \mathrm{~mm}$ lateral, $2.3 \mathrm{~mm}$ posterior, and $4 \mathrm{~mm}$ inferior to the mid-point of the AC-PC line. As can be appreciated from the planned values shown in fig $5 \mathrm{~A}-\mathrm{C}$, the individual values after direct visualisation of the STN on MRI differ considerably from the guideline values of $12 \mathrm{~mm}$ lateral, $3 \mathrm{~mm}$ posterior, and $4 \mathrm{~mm}$ inferior. These variations probably depict the difference in individual anatomy.

In this series, in the initial 12 placements where the STN was less clearly seen, a second track was required in $42 \%$ placements compared with $21 \%$ in the subsequent 42 placements where the STN was defined more effectively. 
Improved MRI specifications and good visualisation of the STN is proving helpful in targeting the STN. It is speculated that with increasing use of MRI and better imaging techniques, intraoperative microrecording may become less essential.

\section{Target localisation: electrophysiology}

In this series, the length of STN activity in 50 recordings $\left(92.6 \%\right.$ ) was $4.65 \mathrm{~mm}$ (range 3-7 mm). Bejjani et al ${ }^{11}$ report good recording in all their patients, with the longest recording of $6-7 \mathrm{~mm}$. In another study, good activity was recorded in $100 \%$ patients with a mean length of $5.4 \mathrm{~mm}$ (range $2.2-6.6 \mathrm{~mm}) .{ }^{10}$

In the present study, the MRI defined target point was "fine tuned" in $90 \%$ of implantations. Most of these adjustments involved alteration of the depth of the electrode. The adjustment was more than $2 \mathrm{~mm}$ in 35\% electrode placements. Some workers have looked into the change of the electrode position quantitatively. Bejjani et al ${ }^{11}$ have reported that in $21 \%$ of their placements, the final electrode locations differed by more than $2 \mathrm{~mm}$ from their initial calculated target before electrophysiological recording. Starr et $\mathrm{al}^{3}$ have reported a difference of greater than $2 \mathrm{~mm}$ in $19 \%$ of placements. Intraoperative electrophysiology led to adjustment of the target in a significant number of implantations in other reported series. ${ }^{717}$

A discrepancy between targets defined by various stereotactic imaging methods and electrophysiologically determined targets has been observed by various workers and many possible explanations have been put forward, one of which is that the determination of the target coordinates is not very accurate. ${ }^{78}$ Another is MR image distortion ${ }^{19}$ and brain shifts after the initial images have been obtained have also been blamed. ${ }^{30}$ In this series, the side operated first required less electrophysiological mapping to get a good STN recording. Cerebrospinal fluid leakage occurring during the time interval to operating on the second side may be responsible for brain shifts. Electrophysiology probably provides an objective measure of these errors in real time.

The usefulness and even the need for electrophysiology are still debated. Some groups have reported good outcomes without intraoperative recording. ${ }^{16}$

\section{Trajectory}

The trajectory or approach angle used to reach the STN varies considerably among surgeons. This has an implication on electrophysiological recordings as the area being recorded may vary. Compared with our mean angle of $17.5^{\circ}$ in the coronal projection, Bejjani $e t$ al ${ }^{11}$ used a slightly more lateralto-medial angle of $20-30^{\circ}$ from the vertical. On the other hand, Starr et $a l^{3}$ used a more medial approach with a mean angle of $10.2^{\circ}$. Our average angle in the sagittal projection was $38^{\circ}$ from the vertical plane. Rodriguez et al ${ }^{17}$ report a similar horizontal trajectory with an angle of $45^{\circ}$, while a more vertical trajectory with a mean angle of $29.2^{\circ}$ has been used by another team. ${ }^{3}$ These values are also significant in calculating the amount of movement in the different axes if the depth of the electrode is altered.

\section{Final target location}

In our study, the mean of the final target used for implantation (after electrophysiological recordings and correction) was 11.7 (1.30) mm lateral, 2.1 (1.43) mm posterior, and $3.8(1.22) \mathrm{mm}$ inferior to the centre of the AC-PC line. In one study using MRI localisation and electrophysiology, mean coordinates of the active contacts were $11.8 \mathrm{~mm}$ lateral, $2.4 \mathrm{~mm}$ posterior, and $3.7 \mathrm{~mm}$ inferior to the centre of the AC-PC line. ${ }^{3}$ These values are very similar to the mean values found in our series. Interestingly, in both this report and our study the final target used was slightly more anterior and superior to the target defined by Benabid et al. ${ }^{9}$ It must be realised that individual target locations varied considerably. Therefore, fixed reference values cannot be recommended for use in all patients indiscriminately. However, it has been pointed out that although the placement of the stimulating electrode into the centre of the STN produces the best clinical outcome, adjacent structures may be used as alternative targets. ${ }^{21}$

\section{Postoperative MRI}

The safety of MRI with deep brain stimulators has always been questioned. In addition to the 16 cases presented in this study, another 19 patients with DBS have undergone postoperative MRI in our unit. The manufacturer of these devices (Medtronic) continues to caution against the routine use of postoperative MRI. Many radiologists are understandably reluctant. Despite these pitfalls, many workers use it for verifying lead position. ${ }^{3}{ }^{12} 22$ None of these groups has described any adverse effects. We used head coils and a 1.5 Tesla magnet. These parameters have been found to be safe. ${ }^{23}{ }^{24}$ The electrode produces an artefact that is bigger than its actual diameter. The centre of this artefact was assumed to be the most representative point. Though the same assumption has been used for calculations by other workers, ${ }^{325}$ there is no definitive proof that the actual contact is located in the centre of the artefact. However, in a recent study where this issue was addressed specifically, it was found that that the average diameter of the electrode artefact was $1.5-1.9 \mathrm{~mm}$ (the actual diameter of the lead being $1.27 \mathrm{~mm}$ ) and that the centre of the artefact does actually represent the centre of the electrode. ${ }^{21}$

\section{Error in lead placement}

Various techniques have been used to verify lead location and accuracy of electrode placement. Cuny et $\mathrm{al}^{7}$ used fusion of the preoperative MRI with a postoperative computed tomography scan to define electrode location in relation to the STN. In our series, preoperative MR images (depicting the final target points used after electrophysiological correction) were fused to the postoperative MR images displaying actual electrode location to measure the error in lead placement. An automatic computerised fusion programme matching voxel for voxel was used. This enabled us to see the electrode position in relation to the target point that was used. This is a relatively new technique, so comparative data are lacking in the literature.

In a recent study by Schrader et al ${ }_{1}^{12}$ in which fusion of preand postoperative MRI by landmark coregistration was used, a preliminary review of six cases revealed an error of $0.5 \mathrm{~mm}$ in the $\mathrm{x}$ axis and $2 \mathrm{~mm}$ in the $\mathrm{z}$ axis. Our figures of $0.48 \mathrm{~mm}$, $0.69 \mathrm{~mm}$, and $2.9 \mathrm{~mm}$ in the $\mathrm{x}, \mathrm{y}$, and $\mathrm{z}$ axes, respectively are comparable. In the axial plane, the good electrode placement in the $\mathrm{x}$ and $\mathrm{y}$ axes means that the stereotactic technique using the Leksell-G frame is reasonably accurate. However, the placement was less accurate in the $\mathrm{z}$ axis. Unfortunately, when the accuracy of electrode placement was checked in our initial 16 patients, the technique of fixation of the electrode meant that some electrodes were pushed lower than the target. Some electrodes had to be pulled back a little after checking the position with the image intensifier (see fig 3 ). The finding on MRI that electrodes were slightly lower than the point that was actually targeted, confirms our peroperative anticipated inaccuracy while fixing the electrode in the burr-hole.

\section{CONCLUSION}

Both direct visualisation of the STN on MRI and intraoperative electrophysiological recording are important in defining 
the best target for stimulation. Peroperative electrophysiology defines the STN electrical activity in real time. Because of the fact that there are individual variations in the anatomical location of the STN and the site of best STN electrical activity, use of fixed values for targeting cannot be recommended. In the present series, it was easier to define STN electrical activity on the side operated first, hence it is suggested that the more affected side should be targeted first. The automatic voxel for voxel fusion software enabled us to confirm that the stereotactic electrode placement was accurate especially when measured in the axial plane for the $\mathrm{x}$ and $\mathrm{y}$ axes. Postoperative MRI is a safe and easy way of defining electrode location. Hence, if predictable clinical improvement does not occur, the accuracy of placement can be checked.

\section{ACKNOWLEDGEMENTS}

Our thanks are due to Dr A Sutcliffe who took personal interest in supervising the local and general anaesthesia in various stages of the operations, Mrs J Martey Parkinson's Disease Surgery Nurse Specialist for her support and Mr Michael Port of the University of Sheffield for designing the microdrive used initially for recording.

\section{Authors' affiliations}

N A Hamid, R D Mitchell, P Mocroft, G W M Westby, J Milner, H Pall, Neuroscience Centre, Queen Elizabeth Hospital, Birmingham, UK

Competing interests: none declared

\section{REFERENCES}

1 Benabid AL, Pollak P, Gross C, et al. Acute and long-term effects of subthalamic nucleus stimulation in Parkinson's disease. Stereotact Funct Neurosurg 1994;62:76-84.

2 Pollak P, Benabid AL, Gross C, et al. Effects of the stimulation of the subthalamic nucleus in Parkinson's disease. Rev Neurol (Paris) 1993; 149:175-6.

3 Starr PA, Christine CW, Theodosopoulos PV, et al. Implantation of deep brain stimulators into the subthalamic nucleus: technical approach and magnetic resonance imaging-verified lead locations. J Neurosurg 2002;97:370-87.

4 Pahwa R, Wilkinson SB, Overman J, et al. Bilateral subthalamic stimulation in patients with Parkinson disease: long-term follow up. J Neurosurg 2003;99:71-7.

5 Limousin P, Krack P, Pollak P, et al. Electrical stimulation of the subthalamic nucleus in advanced Parkinson's disease. N Engl J Med 1998:339:1105-11.

6 Bergman H, Wichmann T, DeLong MR. Reversal of experimental parkinsonism by lesions of the subthalamic nucleus. Science 1990;249:1436-8.

7 Cuny E, Guehl D, Burbaud P, et al. Lack of agreement between direct magnetic resonance imaging and statistical determination of a subthalamic target: the role of electrophysiological guidance. J Neurosurg 2002;97:591-7.
8 Limousin P, Pollak P, Benazzouz A, et al. Effect of parkinsonian signs and symptoms of bilateral subthalamic nucleus stimulation. Lancet 1995;345:91-5.

9 Benabid AL, Pollack P, Benazzouz A, et al. Grenoble guidelines for deep brain stimulation, in First European Symposium on Stimulation in Parkinson Disease. Grenoble, France: Universite Joseph Fourier de Grenoble, 1998:13

10 Zonenshayn M, Rezai AR, Mogilner AY, et al. Comparison of anatomic and neurophysiological methods for subthalamic nucleus targeting. Neurosurgery 2000;47:282-92, discussion 292-4.

11 Bejiani BP, Dormont D, Pidoux B, et al. Bilateral subthalamic stimulation for Parkinson's disease by using three-dimensional stereotactic magnetic resonance imaging and electrophysiological guidance. J Neurosurg 2000;92:615-25.

12 Schrader B, Hamel W, Weinert D, et al. Documentation of electrode localization. Mov Disord 2002:167-74.

13 Morel A, Magnin M, Jeanmonod D. Multiarchitectonic and stereotactic atlas of the human thalamus. J Comp Neurol 1997:387:588-630, Erratum in: J Comp Neurol 1998;391:545.

14 Schaltenbrand G, Wahren W. Atlas for Stereotaxy of the Human Brain. New York: Georg Thieme, 1977.

15 Starr PA, Vitek JL, DeLong M, et al. Magnetic resonance imaging-based stereotactic localization of the globus pallidus and subthalamic nucleus. Neurosurgery 1999;44:303-13.

16 Voges J, Volkmann J, Allert N, et al. Bilateral high-frequency stimulation in the subthalamic nucleus for the treatment of Parkinson disease: correlation of therapeutic effect with anatomical electrode position. J Neurosurg 2002;96:269-79

17 Rodriguez MC, Guridi OJ, Alvarez L, et al. The subthalamic nucleus and tremor in Parkinson's disease. Mov Disord 1998;13(suppl 3):111-18.

18 Merello M, Cammarota A, Cerquetti D, et al. Mismatch between electrophysiologically defined and ventriculography based theoretical targets for posteroventral pallidotomy in Parkinson's disease. J Neurol Neurosurg Psychiatry 2000;69:787-91.

19 Sumanaweera TS, Adler JR Jr, Napel S, et al. Characterization of spatial distortion in magnetic resonance imaging and its implications for stereotactic surgery. Neurosurgery 1994;35:696-704.

20 Kirschman DL, Milligan B, Wilkinson S, et al. Pallidotomy microelectrode targeting: neurophysiology-based target refinement. Neurosurgery 2000:46:613-22.

21 Yelnik J, Damier P, Demeret S, et al. Localization of stimulating electrodes in patients with Parkinson disease by using a three-dimensional atlas-magnetic resonance imaging co registration method. J Neurosurg 2003;99:89-99.

22 Saint-Cyr JA, Hoque T, Pereira LC, et al. Localization of clinically effective stimulating electrodes in the human subthalamic nucleus on magnetic resonance imaging. J Neurosurg 2002;97:1152-66.

23 Rezai AR, Lozano AM, Crawley AP, et al. Thalamic stimulation and functional magnetic resonance imaging: localization of cortical and subcortical activation with implanted electrodes. Technical note. J Neurosurg 1999;90:583-90.

24 Tronnier VM, Staubert A, Hahnel S, et al. Magnetic resonance imaging with implanted neurostimulators: an in vitro and in vivo study. Neurosurgery 1999:44:118-25, discussion 125-6.

25 Yelnik J, Damier P, Bejiani BP, et al. Functional mapping of the human globus pallidus: contrasting effect of stimulation in the internal and external pallidum in Parkinson's disease. Neuroscience 2000;101:77-87. 